\title{
BMJ Open Effects of treadmill slip and trip perturbation-based balance training on falls in community-dwelling older adults (STABILITY): study protocol for a randomised controlled trial
}

\author{
Jens Eg Nørgaard (1) ,1,2 Stig Andersen (1) ,1,2 Jesper Ryg (D) ,3,4 \\ Andrew James Thomas Stevenson, ${ }^{5}$ Jane Andreasen, ${ }^{5,6}$ Mathias Brix Danielsen, ${ }^{1}$ \\ Anderson de Souza Castelo Oliveira, ${ }^{7}$ Martin Grønbech Jørgensen ${ }^{1}$
}

To cite: Nørgaard JE, Andersen S, Ryg J, et al. Effects of treadmill slip and trip perturbation-based balance training on falls in community-dwelling older adults (STABILITY): study protocol for a randomised controlled trial. BMJ Open 2022;12:e052492. doi:10.1136/ bmjopen-2021-052492

- Prepublication history and additional supplemental material for this paper are available online. To view these files, please visit the journal online (http://dx.doi.org/10.1136/ bmjopen-2021-052492)

Received 19 April 2021 Accepted 11 January 2022

Check for updates

(C) Author(s) (or their employer(s)) 2022. Re-use permitted under CC BY-NC. No commercial re-use. See rights and permissions. Published by BMJ.

For numbered affiliations see end of article.

Correspondence to

Jens Eg Nørgaard;

jens.noergaard@rn.dk

\section{ABSTRACT}

Introduction Falls among older adults are most frequently caused by slips and trips and can have devastating consequences. Perturbation-based balance training (PBT) have recently shown promising fall preventive effects after even small training dosages. However, the fall preventive effects of PBT delivered on a treadmill are still unknown. Therefore, this parallel-group randomised controlled trial aims to quantify the effects of a four-session treadmillPBT training intervention on falls compared with treadmill walking among community-dwelling older adults aged 65 years or more.

Methods and analysis 140 community-dwelling older adults will be recruited and randomised into either the treadmill-PBT or the treadmill walking group. Each group will undergo three initial training sessions within a week and an additional 'booster' session after 26 weeks. Participants in the treadmill-PBT group will receive 40 slip and/or trip perturbations induced by accurately timed treadmill belt accelerations at each training session. The primary outcome of interest is daily life fall rates collected using fall calendars for a follow-up period of 52 weeks. Secondary outcomes include physical, cognitive and social-psychological fall-related risk factors and will be collected at the pre-training and post-training test and the 26-week and 52-week follow-up tests. All outcomes will be analysed using the intention-to-treat approach by an external statistician. A Poisson's regressions with bootstrapping, to account for overdispersion, will be used to compare group differences in fall rates.

Ethics and dissemination The study protocol has been approved by the North Denmark Region Committee on Health Research Ethics (N-20200089). The results will be disseminated in peer-reviewed journals and at international conferences.

Trial registration number NCT04733222.

\section{INTRODUCTION}

Slips and trips accounts for $60 \%$ of accidental falls among older adults and often leads to serious consequences such as disability,

\section{Strengths and limitations of this study}

- Large-scale randomised controlled trial, powered to determine the effect of treadmill perturbation-based balance training (treadmill-PBT) on daily life fall rates among older adults ( $65+$ years of age).

- Assessor-blinded, prospective, 52-week daily life fall data monitoring using fall calendars as recommended by ProFaNE (Prevention of Falls Network Europe).

- Effects of treadmill-PBT will be explored on numerous fall-related risk factors and important sociopsychological aspects.

- Blinding of participants will not be possible, potentially leading to response bias.

- The assessor of the secondary fall risk factors will not be blinded, which could introduce observer bias.

institutionalisation, decreased quality of life and premature death. ${ }^{1-4}$ Annually medical costs related to falls are high and is estimated to account for $\sim 1 \%$ of Danish total health expenditure $\left(\sim € 200\right.$ million in 2016). ${ }^{5}$ Fallrelated medical costs are expected to grow as the older population will increase in numbers in the upcoming decades. Thus, effective fall prevention interventions are warranted to improve the well-being of older adults and reduce future medical expenses for society. ${ }^{6-9}$

A comprehensive systematic review from 2020, assessing 64 randomised controlled trials (RCTs), showed that general exercise decreased fall rates by $23 \% .^{10}$ This review, among others, have paved the road for conventional exercises such as balance training and strength training to become central aspects of fall preventive recommendation worldwide. ${ }^{811}$ While these conventional exercise regimens target specific fall risk factors, the 
principle of task-specificity suggests approaches that directly address the fall-related context may provide better fall prevention. ${ }^{12}{ }^{13}$ Perturbation-based balance training (PBT), in which participants are exposed to repeated slips and trips in a safe environment, could be one such approach. Among older adults, PBT has been shown to improve proactive and reactive dynamic stability resulting in a reduced risk of falling after a laboratory-induced slip and trip perturbation by 50\%-100\%. ${ }^{12-18}$ Additionally, two meta-analyses from 2015 and 2017, which assessed eight and four studies, showed that daily life fall rates decreased by $46 \%$ and $48 \%$ after PBT, respectively. ${ }^{19} 20$

PBT adaptations have interestingly been observed after as little as a single session and appear to be maintained for up to 12 months. ${ }^{14} 15$ 21-24 In contrast, conventional exercise regimes demands continuous weekly participation to preserve the fall preventive effect. ${ }^{25}$ Furthermore, poor compliance with such exercise approaches is often reported, which inevitably causes meagre long-term prevention. ${ }^{2627}$ The brevity of PBT reduce the reliance on continuous self-motivation and potentially promotes the older adults' willingness to conduct the necessary amount of training. ${ }^{12}$ PBT may, therefore, emerge as an effective, sustainable and relatively inexpensive fall prevention intervention.

PBT has previously been performed using a variety of methods such as; (1) movable platforms, ${ }^{28}$ (2) walkways with low friction platforms and/or trip boards ${ }^{1416}$ and (3) treadmill that produces sudden accelerations. ${ }^{29-32}$ Among these, approaches that apply perturbations during walking are considered more task-specific as most falls occur during walking. ${ }^{33}$ Moreover, perturbations delivered on walkways are considered the most realistic; however, such setups is space-consuming, expensive and immobile. Treadmills, conversely, are less spaceconsuming, cheaper and more portable, enabling a more straightforward implementation into fall prevention and rehabilitation clinics. ${ }^{12} 293536$ An additional benefit of treadmills is the perturbations' unpredictability, which enhances the reliance on reactive balance control strategies. ${ }^{23}$ Studies have shown that treadmill-PBT improves proactive and reactive dynamic stability and decreases fall rates following laboratory-induced walkway perturbations. ${ }^{29} 30353738$ Nonetheless, the magnitude to which these adaptations translates to prevent daily life falls are still vastly unknown. ${ }^{31}$ Rosenblatt $e t$ al showed that four 1-hour sessions of trip treadmill-PBT decreased the rate of daily life trip-related falls by $46 \%(95 \% \mathrm{CI}=3 \%$ to $70 \%$ ) in 210 women aged 55 or more. ${ }^{31}$ The training did, however, not lead to any differences in overall fall rates. ${ }^{31}$ The treadmill-PBT protocol used by Rosenblatt et al only consisted of trip perturbations applied in a standing position, which might explain the absent fall preventive effect since people most often fall while moving. ${ }^{31}$ More recently, ${ }^{32}$ Lurie et al investigated, in a highly pragmatic RCT, the effects of adding treadmill-PBT using both trip and slip perturbations to usual multimodal exercise-based balance training at an outpatient physical therapy clinic. ${ }^{32}$ While the number of injurious falls was decreased after 3 months, no differences in daily life fall rates were found among the 506 older adults. ${ }^{32}$ However, the pragmatic nature of the study by Lurie $e t$ al prevents a standardised dose and intensity of the treadmill-PBT protocol limiting the ability to draw conclusions. ${ }^{32}$ Thus, the current literature limitations highlight the need for large-scale RCTs using treadmill-PBT in multiple directions (slips and trips) and with recommended training doses to elucidate the effects on daily life falls in older adults. ${ }^{23} 39$

Our primary objective of this parallel-group RCT will therefore be to determine the effects of treadmill-PBT on fall rates in community-dwelling older adults aged 65 or older, compared with treadmill walking without perturbations. Second, we aim to evaluate the effects on physical, cognitive and social-psychological fall-related risk factors and the intervention's health economic impact.

\section{METHODS}

\section{Study design and setting}

The effects of treadmill-PBT will be investigated in a parallel-group randomised controlled superiority trial with a 1:1 allocation ratio (see figure 1). The study will be performed as a collaboration between Aalborg University Hospital, Aalborg University and Aalborg Municipality, Denmark. All interventional and testing activities will be conducted in a laboratory placed at Aalborg University. This study protocol follows the Standard Protocol Items: Recommendations for Interventional Trials guidelines, ${ }^{40}$ and has been registered on ClinicalTrials.gov.

\section{Participants}

We aim to recruit 140 community-dwelling older adults (70 in each group) living in and around Aalborg via advertisements in local and national newspapers, radio and television spots and snowball sampling. Participants are included if they are (1) $\geq 65$ years old, (2) communitydwelling and (3) able to walk without a walking aid. Participants will be excluded if they (1) have any of the following self-reported conditions: orthopaedic surgery within the past 12 months, osteoporosis or history of osteoporosis-related fractures (low-impact hip, spine and wrist fracture) or progressive neurological disease (eg, Parkinson's disease), (2) have an unstable medical condition that would prevent safe participation, (3) have a severe cognitive impairment (a score $<8$ in The Short Orientation-Memory-Concentration Test) ${ }^{41}$ and (4) are currently participating in another fall prevention trial.

Potential participants will receive written information about the study followed by verbal information from a research staff member over the phone. During the phone call, the research staff will also screen for eligibility, obtain verbal consent and arrange an initial session appointment. 
Recruitment

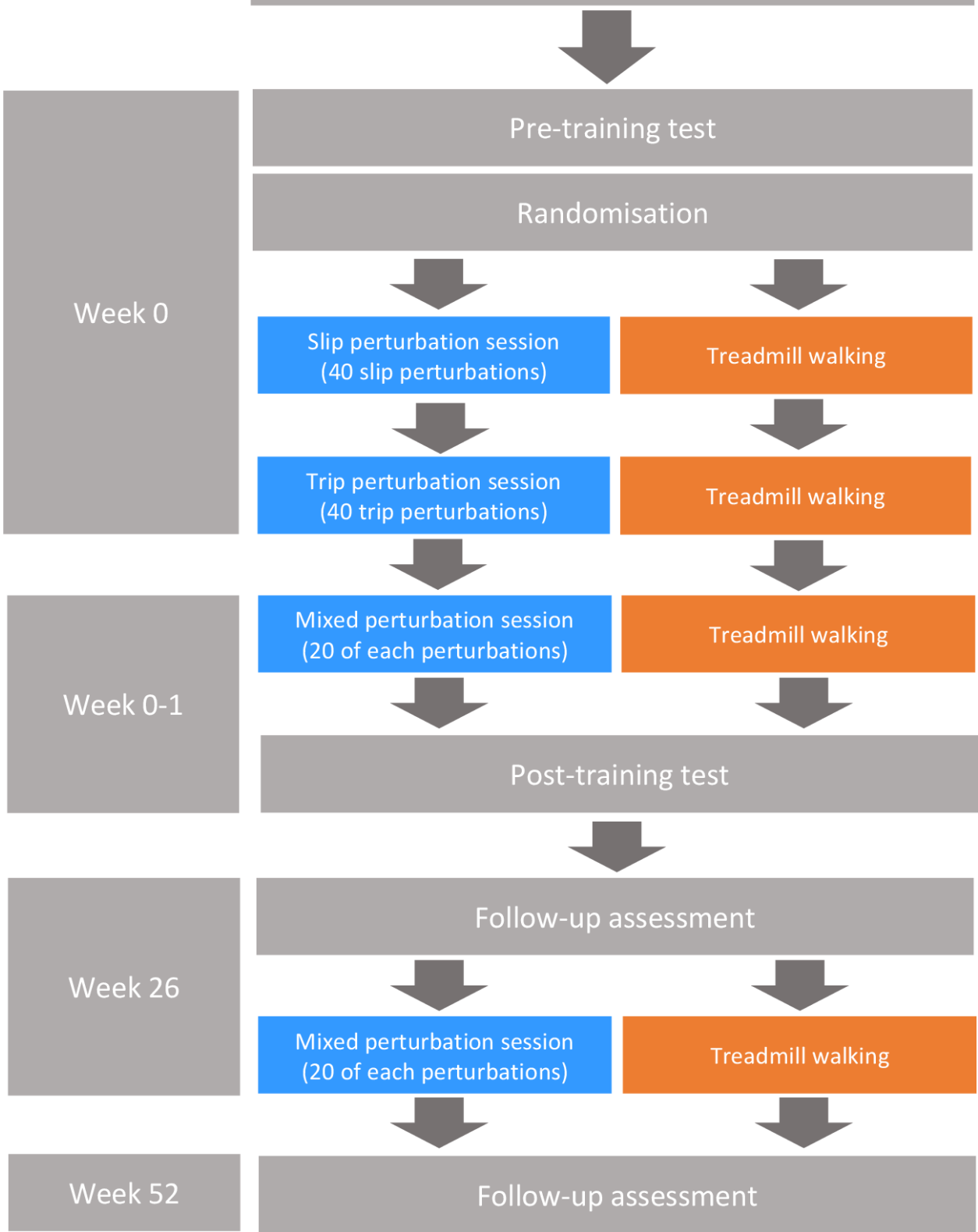

Figure 1 Illustration of the study flow. Blue squares indicate the study flow of the treadmill-perturbation-based balance training group, while the orange squares illustrate the study flow of the treadmill walking group.

\section{Randomisation}

After the pre-training tests, participants will be randomly allocated to either the treadmill-PBT or treadmill walking group using a randomisation module in REDCap (Research Electronic Data Capture; V.9.5.6). Permuted block randomisation will be used to produce similar group sizes, and random block sizes (two, four, six or eight) will ensure that allocation concealment is maintained. The allocation sequence will be generated by a research staff member not involved in enrolling or assigning participants to groups. A timeline for enrolment, intervention and assessment of the participants is presented in table 1 .

\section{Interventions}

Before the first training session, all participants will walk for $5 \mathrm{~min}$ at $50 \%$ of their overground walking speed to familiarise themselves with the treadmill. ${ }^{42}$ The preferred treadmill walking speed will then be determined by gradually increasing and decreasing the treadmill speed to identify the participant's upper and lower boundaries of comfortable walking. The mean velocity of these boundaries will be defined as the preferred walking speed. ${ }^{434}$ The preferred walking speed found during the first training session will be used for all training sessions.

A thorough description of the treadmill-PBT and treadmill walking intervention, following the Template for 
Table 1 Schedule for enrolment, intervention and assessment

\section{Study period}

\begin{tabular}{|c|c|c|c|c|c|c|}
\hline & Enrolment & Pre-training & Intervention & Post-training & 26-week follow-up & 52-week follow-up \\
\hline Time point & $-\mathrm{T} 1$ & TO & T1 & T2 & T3 & T4 \\
\hline \multicolumn{7}{|l|}{ Enrolment: } \\
\hline Informed written consent & & $x$ & & & & \\
\hline Randomised allocation & & $\mathrm{x}$ & & & & \\
\hline \multicolumn{7}{|l|}{ Treadmill-PBT } \\
\hline \multicolumn{7}{|l|}{ Walking training } \\
\hline \multicolumn{7}{|l|}{ Assessments: } \\
\hline \multicolumn{7}{|l|}{ Falls } \\
\hline Neurophysiological & & $x$ & & $x$ & $x$ & \\
\hline Descriptive & & $X$ & & & & \\
\hline
\end{tabular}

PBT, perturbation-based balance training

Intervention Description and Replication guidelines, is provided in online supplemental material $1 .{ }^{40}$ Participants in both the treadmill-PBT and treadmill walking group will be encouraged to continue their regular activity schedule during the trial period.

\section{Treadmill-PBT}

The treadmill-PBT group will be assigned to four training sessions in total. The two initial training sessions will be performed on the same day, separated by an approximate 5 min break, and will consist of only slip (first session) and trip (second session) perturbations. These sessions, with predictable perturbation types, are planned to enhance the participant's confidence and decrease anxiety associated with training. ${ }^{145}$ The third session is performed a week later and will consist of randomly ordered slip and trip perturbations, which have been shown to maximise the training effects. ${ }^{46}$ Lastly, the fourth session will be similar to the third session but performed at week 26 and will serve as a booster training, which previously has been shown to sustain training effects for longer. ${ }^{48}$ An overview of the study flow is provided in figure 1 .

Each training session will have a duration of approximately $20 \mathrm{~min}$, and consist of 40 perturbations delivered bilaterally with 20 perturbations to each leg in random order on a computer-controlled treadmill moving uniformly (Split 70/157/ASK; Woodway, Weil am Rhein, Germany). To further enhance the unpredictability of the perturbations, the duration (10-50 steps) between each perturbation will be random. Before the first perturbation, participants will be shown a video of both the slip and trip perturbation to minimise potential anxiety associated with the situation. An overhead harness safety system will secure the participants and prevent them from falling to the ground during training.

The slip perturbations are induced by a quick forward acceleration at heel strike ( $0 \%$ of the gait cycle), causing a reversal in the direction of the treadmill, resulting in a backward loss of balance. The trip perturbations are caused by a slight deceleration followed by a large backward acceleration of the treadmill during the mid-swing phase $(\sim 80 \%$ of the gait cycle), causing a forward loss of balance. The perturbation intensity for each of the training sessions will be adjusted to the participants preferred walking speed and will be divided into five levels with progressively longer perturbation durations (slips) or greater accelerations (trips) (see table 2). The protocol is split into 11 blocks of two to four perturbations arranged in a progressive ascending-mixedintensity manner (see figure 2). ${ }^{30} 49$ The ascending phase serves as a warm-up and to increase the training's tolerability, while the mixed phase facilitates overlearning to maximise the training effects. ${ }^{49}$ After each block, the participants will rate their perceived difficulty and anxiety on a Visual Analogue Scale from 1 to 5. For the intensity to be increased, the following three criteria have to be met: (1) the combined perceived difficulty and anxiety score have to be 4 or less, (2) the participant did not fall during any of the perturbations in the block prior and (3) the participants accepted to increase the intensity. If any of these criteria are not met, the intensity remains unaltered. 
Table 2 Schematic presentations of the intensity levels in the slip perturbation protocol $(A)$ and the trip perturbation protocol (B)

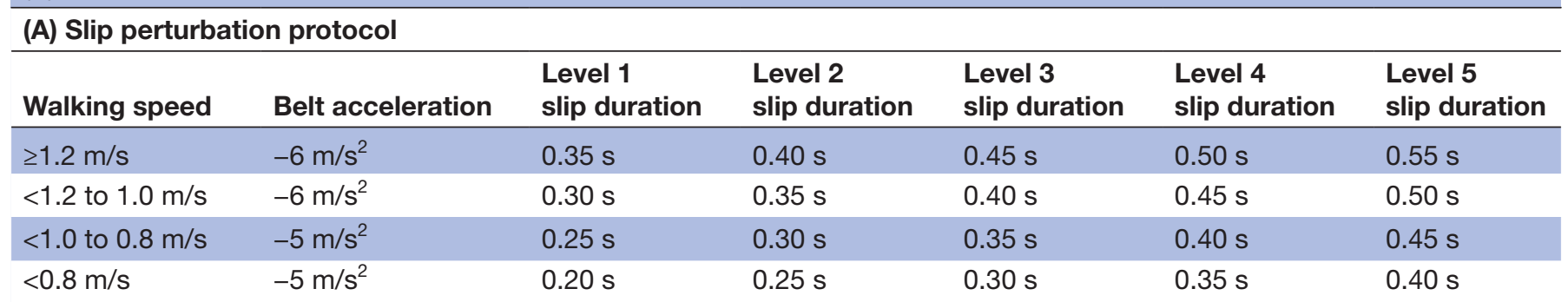

(B) Trip perturbation protocol

\begin{tabular}{llllll}
\hline Walking speed & $\begin{array}{l}\text { Level 1 } \\
\text { trip acceleration }\end{array}$ & $\begin{array}{l}\text { Level 2 } \\
\text { trip acceleration }\end{array}$ & $\begin{array}{l}\text { Level 3 } \\
\text { trip acceleration }\end{array}$ & $\begin{array}{l}\text { Level 4 } \\
\text { trip acceleration }\end{array}$ & $\begin{array}{l}\text { Level } 5 \\
\text { trip acceleration }\end{array}$ \\
\hline$\geq 1.2 \mathrm{~m} / \mathrm{s}$ & $7 \mathrm{~m} / \mathrm{s}^{2}$ & $8 \mathrm{~m} / \mathrm{s}^{2}$ & $9 \mathrm{~m} / \mathrm{s}^{2}$ & $10 \mathrm{~m} / \mathrm{s}^{2}$ & $11 \mathrm{~m} / \mathrm{s}^{2}$ \\
$<1.2$ to $1.0 \mathrm{~m} / \mathrm{s}$ & $6 \mathrm{~m} / \mathrm{s}^{2}$ & $7 \mathrm{~m} / \mathrm{s}^{2}$ & $8 \mathrm{~m} / \mathrm{s}^{2}$ & $9 \mathrm{~m} / \mathrm{s}^{2}$ & $10 \mathrm{~m} / \mathrm{s}^{2}$ \\
$<1.0$ to $0.8 \mathrm{~m} / \mathrm{s}$ & $5 \mathrm{~m} / \mathrm{s}^{2}$ & $6 \mathrm{~m} / \mathrm{s}^{2}$ & $7 \mathrm{~m} / \mathrm{s}^{2}$ & $8 \mathrm{~m} / \mathrm{s}^{2}$ & $9 \mathrm{~m} / \mathrm{s}^{2}$ \\
$<0.8 \mathrm{~m} / \mathrm{s}$ & $4 \mathrm{~m} / \mathrm{s}^{2}$ & $5 \mathrm{~m} / \mathrm{s}^{2}$ & $6 \mathrm{~m} / \mathrm{s}^{2}$ & $7 \mathrm{~m} / \mathrm{s}^{2}$ & $8 \mathrm{~m} / \mathrm{s}^{2}$ \\
\hline
\end{tabular}

\section{Treadmill walking}

Participants allocated to the treadmill walking group will undergo four training sessions arranged similar to the treadmill-PBT group. Each training session consists of 20 min of treadmill walking, matching the duration spent on the treadmill by the treadmill-PBT group.

\section{Outcomes}

The participants' descriptive data will be collected following recommendations on conducting and reporting trials in older adults during the pre-training tests. ${ }^{50}$ Descriptive data include height, weight, sex, physical and cognitive function, medication usage, Tilburg Frailty Indicator, highest education level, living arrangements, fall history including associated injuries, Vulnerable Elders Survey-13 (everyday activity functionality), physical activity levels and home care usage. Information will be collected through a combination of self-reporting, measurements, questionnaires and medical/municipality records (see table 3 ).

\section{Primary outcome}

The primary outcome is daily life fall rate (falls per person-year). Daily life falls will be assessed continuously throughout a 52-week period using fall calendars as recommended by ProFaNE. ${ }^{51}$ The fall calendars are designed for daily recordings and monthly returns by mail in prestamped envelopes. A fall is defined as 'an unexpected event in which the participant comes to rest on the ground, floor or lower-level'. ${ }^{51}$ When a fall has occurred, a research staff member blinded to group allocation will call the participant. During this phone interview, information about the fall's circumstances and consequences (eg, fall-related injuries) will be obtained. If a fall calendar is not returned within 14 days from the deadline, participants will be contacted to acquire the missing information.

\section{Secondary outcomes}

The secondary outcomes include other fall metrics, physical, cognitive and social-psychological measures, which will elucidate the effects of the treadmill-PBT intervention on important fall risk factors. An overview of the tests and timing of the assessments can be seen in table 3 .

Secondary fall and fall-related injury metrics will also be collected via the aforementioned fall calendars. The fall metrics include (1) the number of participants with at least one fall and (2) the time to first fall. Fall-related injury metrics include (1) the number of fracture events

Ascending phase

Mixed phase Cool-down phase

\begin{tabular}{|c|c|c|c|c|c|c|c|c|c|c|}
\hline $4 x$ & $4 x$ & $4 x$ & $4 x$ & $4 x$ & $4 x$ & $4 x$ & $4 x$ & $4 x$ & $2 x$ & $2 x$ \\
Level 1 & Level 2 & Level 3 & Level 4 & Level 5 & Level 4 & Level 5 & Level 4 & Level 5 & Level 3 & Level 1 \\
\hline
\end{tabular}

\section{0 total perturbations}

Figure 2 The sequential arrangement of perturbation intensity levels in the training protocol. The protocol is arranged in three phases: (1) an ascending phase in which the intensity of the perturbations progressively increases, (2) a mixed phase where the perturbation intensity varies between level 4 and 5 and (3) a cool-down phase at which the perturbation intensity decreases. 
Table 3 Assessment of outcomes across the study timeline

\section{Fall assessments}

$\begin{array}{lllll}\begin{array}{l}\text { Pre-training } \\ \text { (T0) }\end{array} & \begin{array}{l}\text { Post- } \\ \text { training } \\ \text { (T2) }\end{array} & \begin{array}{l}\text { 26-week } \\ \text { follow-up } \\ \text { (T3) }\end{array} & \begin{array}{l}\text { 52-week } \\ \text { follow-up } \\ \text { (T4) }\end{array} & \begin{array}{l}\text { Cos } \\ \text { as } \\ \text { (T0- }\end{array} \\ & & & & X \\ & & & & X \\ X & & X & X & X \\ \end{array}$

Falls*

Fall-related injuries

Fall-related use of healthcare services $\dagger$

Laboratory-induced falls $†$

26-week 52-week Continuous

Physical and cognitive assessments

\begin{tabular}{|c|c|c|c|c|c|}
\hline & Pre-training & Post-training & $\begin{array}{l}\text { 26-week } \\
\text { follow-up }\end{array}$ & $\begin{array}{l}\text { 52-week } \\
\text { follow-up }\end{array}$ & $\begin{array}{l}\text { Continuous } \\
\text { assessment }\end{array}$ \\
\hline Single-task and dual-task gait patterns $†$ & $\mathrm{X}$ & $\mathrm{X}$ & $\mathrm{X}$ & $X$ & \\
\hline Choice stepping reaction test $†$ & $x$ & $x$ & $x$ & $x$ & \\
\hline The Short Physical Performance Battery† & $\mathrm{x}$ & $\mathrm{x}$ & $\mathrm{x}$ & $\mathrm{x}$ & \\
\hline The Trail-Making-Test Part A and B† & $\mathrm{X}$ & $\mathrm{X}$ & $\mathrm{X}$ & $X$ & \\
\hline
\end{tabular}

\section{Questionnaire-based assessments}

\begin{tabular}{|c|c|c|c|c|c|}
\hline & Pre-training & Post-training & $\begin{array}{l}\text { 26-week } \\
\text { follow-up }\end{array}$ & $\begin{array}{l}\text { 52-week } \\
\text { follow-up }\end{array}$ & Continuous assessment \\
\hline EQ-5D-5L† & $x$ & $\mathrm{X}$ & $x$ & $\mathrm{X}$ & $\mathrm{X}$ \\
\hline The Tilburg Frailty Index $\ddagger$ & $x$ & & & & \\
\hline Vulnerable Elders Survey-13ł & $x$ & & & & \\
\hline
\end{tabular}

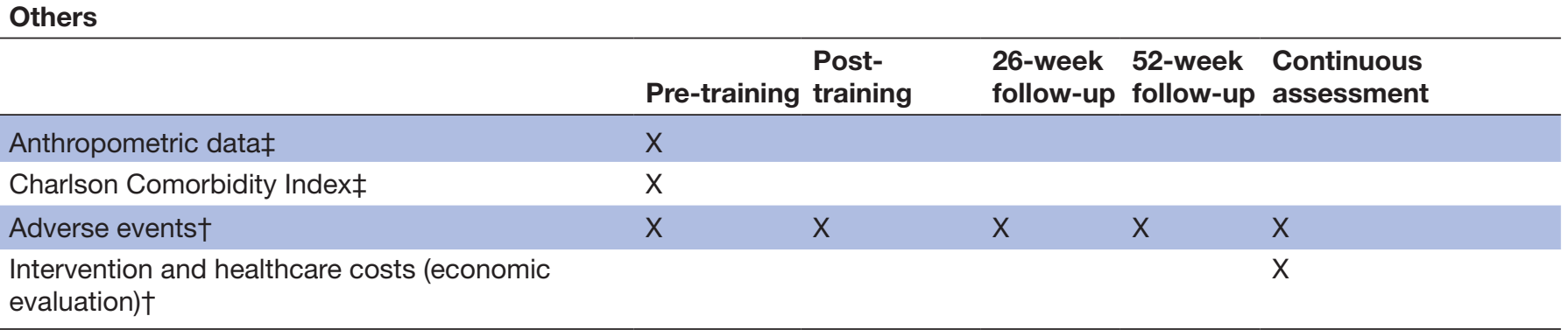

*Fall rate (fall per person-year) is the primary outcome.

†Secondary outcome.

fDescriptive data.

EQ-5D-5L, EuroQol 5-dimensions 5-levels.

per person-year, (2) the number of participants with at least one fracture, (3) the number of other injuries (eg, sprains, bruises and head injuries) per-person year, (4) the number of participants with at least one other injury and (5) the number of fall-related hospital contacts and general practitioner visits. Moreover, at the four testing sessions, a level 1 slip and trip perturbation will be induced to investigate the participants' reactive balance adaptations. The slip and trip perturbations will be recorded in slow motion, and a research staff member, blinded for group allocation, will review the videos to determine if the participant falls or not. It will be deemed a fall if the safety harness unambiguously supports the participant. If the participant does not fall, the number of compensatory steps to regain balance will be assessed.

An unblinded research staff member will conduct the physical assessments that include single-task and dual-task gait patterns, single-task and dual-task static balance, stepping reactions and lower extremity performance. These assessments have been selected because they are identified as risk factors for falls. ${ }^{52-55}$ To assess gait patterns, the participants will be asked to walk 8 metres at their habitual 
pace six times. First, the participants will be instructed to walk for three trials as a single task. They will then be instructed to walk three trials while counting backwards in intervals of three from a random three-digit number as a dual-task. ${ }^{567}$ Participants will not be instructed to prioritise gait over cognitive tasks or vice versa as this provides the best representation of what happens naturally. ${ }^{58}$ The middle 6 metres will be timed and recorded following the recommendations for gait assessments. ${ }^{58}$ Gait speed will be used for further analysis. Balance will be assessed on a Wii Balance Board (WBB) using the FysioMeter software (FysioMeter, V.1.2.1.4, Denmark). ${ }^{59} 60$ Prior research has shown that WBB provides valid and reliable assessments of the centre of pressure displacements in older adults. ${ }^{61}$ Participants will be instructed to stand as still as possible for $30 \mathrm{~s}$ during three single-task and three dual-task trials. The dual-task involves naming items from the grocery store, and the participants will not be given instructions to prioritise either the balance or cognitive task. The area and speed of the centre of pressure displacements will be used for further analysis. ${ }^{59}$ Stepping reactions will be assessed using a choice stepping reaction test on a WBB using the FysioMeter software. ${ }^{53}$ The WBB has previously shown valid and reliable recordings of stepping reaction time in older adults. ${ }^{62}$ During the choice stepping reaction test, participants will be asked to react as fast as possible to visual clues given on a computer screen by tapping the foot on the correct side of the WBB. The visual clues are provided as a green indicator at a random time (between 1 and $4 \mathrm{~s}$ ) and side (left or right). Seven recordings will be made, and the reaction time from the first six (three from each side) will be used for further analysis. ${ }^{62}$ Lastly, The Short Physical Performance Battery, which has been shown to validly and reliably determine older adults' lower extremity performance and frailty, will be used..$^{55}{ }^{63}$ The Short Physical Performance Battery consists of three elements; balance with three different foot positions, two 4-metre walks and five chair-stands. Each element will be scored based on the performance and the score will be used for further analysis.

Executive function is identified as a cognitive fall-related risk factor and will be assessed using the Trail-MakingTest Part A and B. ${ }^{56} 64$ Part A involves sequentially connecting 25 randomly arranged numbers (1-2-3-..-25) with pencil lines, while Part B encompasses sequentially connecting 25 randomly placed numbers and letters (1-A-2-B-...-12-L) in an alternating manner. ${ }^{64}$ The difference in time-to-complete Part A and B (B-A) will be used in the current study, as this index has been suggested to quantify executive function the best. ${ }^{6566}$ Moreover, the participants' global cognitive function will be evaluated using The Short Orientation-Memory-Concentration Test. $^{67}$

Danish-translated questionnaires will assess social-psychological factors. Health-related quality of life will be quantified using the EuroQoL 5-dimensions 5-levels (EQ-5D-5L), ${ }^{68}$ fear of falling will be assessed using The Short Falls Efficacy Scale International ${ }^{69}$ and the enjoyment of the interventions will be determined by the Physical Activity Enjoyment Scale. ${ }^{70}$ The score derived from these questionnaires will be used for further analysis.

\section{Economic evaluation}

An economic evaluation of the treadmill-PBT intervention will be conducted as both a cost-effectiveness analysis (CEA) and cost-utility analysis (CUA) following the guidelines for conducting and reporting economic evaluation of fall prevention strategies. ${ }^{71}$ In the CEA, the outcome measure will be the difference in the number of falls during the 52-week follow-up period. The outcome measure in the CUA will be quality-adjusted life years (QALY) gained quantified using the utility weight of the EQ-5D-5L. ${ }^{72}$ Cost data will prospectively be collected regarding the training programme (staff salaries and expenses, administration, equipment, rental of premises and overhead) and fall-related healthcare resources (hospital admissions, emergency department visits, general practitioner visits, home-care, rehabilitation and nursing home admissions).

\section{Harms}

Participants will be encouraged to report any minor or major adverse event during the testing procedure or the intervention. Furthermore, anxiety related to the treadmill-PBT intervention will continuously be assessed during the training sessions.

\section{Data management}

All data will be collected and managed using the secure, web-based software platform REDCap hosted at The Region of Northern Denmark. ${ }^{73}$ The data collection forms in REDCap ensure strong data integrity by applying functions that check for mandatory information, data ranges and alerts whenever data violates specific limits. ${ }^{74}$ Paper documents, such as written consent forms, will be stored in a locked cabinet in an area of limited access.

\section{Sample size estimation}

The sample size calculation was conducted in $G^{*}$ power (V.3.1.9.4, University of Kiel, Kiel, Germany) using a Poisson regression model. The calculation was made with certain assumptions $(80 \%$ power, $5 \%$ significance level, $50 \%$ difference in fall rate (favouring the PBT) and $20 \%$ dropout rate) and an expected average fall rate of $0.85 .{ }^{75}$ The sample size calculation estimated an required sample size of 70 participants in each group.

\section{Statistical methods}

All statistical analyses will be conducted using the intention-to-treat principle. A per-protocol analysis will also be performed, including only participants who complete $75 \%$ of the training sessions. The statistical analyses will be conducted by an external statistician. The level of significance will be set at $5 \%(\mathrm{p}<0.05)$.

Descriptive data will be presented as mean and SD, median and IQR or number and percentage, where appropriate. Group differences in baseline values will 
be compared using unpaired t-tests for continuous variables, Fisher's exact test for binary variables and Poisson's regression for count variables.

\section{Primary outcome}

The primary outcome, daily life fall rate (count variable), will be analysed using a Poisson's regression with, if necessary, bootstrapping to account for the often-observed overdispersion in fall rate data. ${ }^{76}$ Sensitivity analysis will be made in which we adjust for confounders, including age, sex and previous falls.

\section{Secondary outcomes}

Besides the secondary fall metrics, the secondary outcomes will be analysed as differences in means from the pre-training test to the post-training test, the 26-week and the 52-week follow-up, respectively. Dichotomous outcomes will be analysed using Fisher's exact test. Count outcomes will be analysed using Poisson's regression with bootstrapping if data are overdispersed. Continuous outcomes will be analysed using a 2 (group) $\times 4$ (time) analysis of variance, with repeated measures on the second factor (time). If continuous variables violate the assumption of normal distribution, log-transformation will be performed and, if necessary, bootstrapping. Sensitivity analyses adjusting for age, sex and previous falls will be conducted for all the secondary outcomes.

In the economic evaluation group differences in falls and QALYs are divided by the group-difference in costs to determine the incremental cost-effectiveness ratio of the CEA and CUA's. The $95 \%$ CI will be estimated with bootstrapping, and the result of the economic analysis will be presented on a cost-effectiveness plane. To systematically account for the economic evaluation's uncertainties, sensitivity analyses using the one-way scenario method will be conducted. Additionally, probabilistic sensitivity analysis by making 10000 computer-based Monte-Carlo simulations will be performed and presented on a costeffectiveness acceptability curve to guide the decisionmaking process.

\section{PUBLIC AND PATIENT INVOLVEMENT}

Before recruitment began, pilot trials were conducted on community-dwelling older adults to evaluate the training protocol; however, none of the feedback insinuated any changes to the protocol. The public has been involved in the recruitment process through public service mentions in national television news, local radio and web articles. Neither the public nor patients will be involved in conducting or measuring outcomes. The patients will not assess the burden of the intervention. Written reports of the results will be sent by email to the participants of the trial.

\section{DISCUSSION}

The population of older adults at high risk of falling is expected to increase in the coming decades, highlighting the need for effective fall prevention strategies. ${ }^{6}$ Physical exercise is currently considered an effective method to decrease fall rates; yet, conventional exercise approaches have issues with a high degree of attrition, limiting the long-term prophylactic effect. ${ }^{26} 27757778$ In contrast, PBT has previously shown a vaccination-like effect as even small dosages have been shown to decrease daily life fall rates by $~ 50 \% .{ }^{142339}$ Thus, current pieces of evidence indicates that such training could be an effective, inexpensive and sustainable fall prevention strategy.

In this RCT, we seek to evaluate a four-session treadmill-PBT intervention's effectiveness in reducing the daily life fall rate among older adults aged 65 years or older. The current study's secondary outcomes will also enlighten the effects on well-known fall-related physical and cognitive risk factors. Furthermore, fear of falling and health-related quality of life assessments will elucidate additional transfer effects of treadmill-PBT to other important factors. We believe this study has the potential to decrease the number of daily life falls and fall-related injuries and improve crucial social-psychological factors among older adults. Improvements in any of these parameters offer valuable insights into the beneficial effects of treadmill-PBT targeting older adults. This study will also attempt to address the subsequent implementation as the economic analysis evaluates the intervention's costeffectiveness and -utility. The recruited older participants are expected to be relatively healthy, motivated and community-dwelling; thus, the results will mainly apply to this population.

Recruitment of participants is expected to begin in April 2021, and the results are expected to be accessible in late 2022.

\section{ETHICS AND DISSEMINATION}

The protocol has been approved by The North Denmark Region Committee on Health Research Ethics (N-20200089) and the Danish Data Protection Agency (2021-014). Serious adverse events will be reported within 2 weeks with comments on the participants' safety and potential consequences for the trial. Such events will be reviewed by The North Denmark Region Committee on Health Research Ethics independent from the trial investigators. Additional adverse events will be collected and reported to the local ethics committee annually. The trial participants will be covered by the Danish Act on the Right to Complain and Receive Compensation in healthcare. Furthermore, each participant will provide written informed consent before the commencement of any study activities (online supplemental material 2).

Regardless of the outcome, the results will be disseminated in relevant peer-reviewed scientific journals and at national and international conferences. To facilitate the subsequent implementation of treadmill-PBT at 
fall clinics, the results will be presented to the decisionmakers across the country's municipalities and hospitals. Press releases in layman's terms will be administrated to local and national newspapers, radio and television stations to address the general public, including the use of social media.

Authorship will be determined following the Vancouver Convention. All authors will have provided substantial intellectual contributions to the development of the protocol, the conduct of the study and/or the manuscript. The authors have approved the final manuscript and agree to be accountable for the work.

\section{Author affiliations}

${ }^{1}$ Department of Geriatric Medicine, Aalborg University Hospital, Aalborg, Denmark ${ }^{2}$ Department of Clinical Medicine, Aalborg University, Aalborg, Denmark ${ }^{3}$ Department of Geriatric Medicine, Odense University Hospital, Odense, Denmark ${ }^{4}$ Department of Clinical Research, University of Southern Denmark, DK-5000 Odense, Denmark

${ }^{5}$ Department of Health, Science and Technology, Aalborg University, Aalborg, Denmark

${ }^{6}$ Department of Occupational Therapy and Physiotherapy, Aalborg University Hospital, Aalborg, North Denmark Region, Denmark

${ }^{7}$ Department of Materials and Production, Aalborg University, Aalborg, Denmark

Contributors Author contributions are described using the CRediT taxonomy. JEN: conceptualisation, methodology, developing the formal analysis plan, project administration, software, supervision, visualisation and writing the original draft. SA: conceptualisation, funding acquisition and reviewing/editing the draft. JR: conceptualisation, methodology, visualisation and reviewing/editing the draft. JA: methodology, visualisation and reviewing/editing the draft. AdSC0: methodology, visualisation and reviewing/editing the draft. AJTS: conceptualisation, methodology, software, resources, supervision, visualisation and reviewing/editing the draft. MBD: conceptualisation, methodology, visualisation and reviewing/editing the draft. MGJ has been involved in conceptualisation, methodology, developing the formal analysis plan, funding acquisition, supervision, visualisation and reviewing/editing the draft.

Funding The study is part of a PhD funded by Aalborg Municipality (grant number: N/A), Aalborg University (grant number: N/A) and Department of Geriatric Medicine at Aalborg University Hospital (grant number: N/A).

Competing interests MGJ is a shareholder in FysioMeter, the software used for the balance and stepping reaction assessments.

Patient consent for publication Not applicable.

Provenance and peer review Not commissioned; externally peer reviewed.

Data availability statement As this article is a study protocol, no data will be generated; thus, no data will be available to be shared.

Supplemental material This content has been supplied by the author(s). It has not been vetted by BMJ Publishing Group Limited (BMJ) and may not have been peer-reviewed. Any opinions or recommendations discussed are solely those of the author(s) and are not endorsed by BMJ. BMJ disclaims all liability and responsibility arising from any reliance placed on the content. Where the content includes any translated material, BMJ does not warrant the accuracy and reliability of the translations (including but not limited to local regulations, clinical guidelines, terminology, drug names and drug dosages), and is not responsible for any error and/or omissions arising from translation and adaptation or otherwise.

Open access This is an open access article distributed in accordance with the Creative Commons Attribution Non Commercial (CC BY-NC 4.0) license, which permits others to distribute, remix, adapt, build upon this work non-commercially, and license their derivative works on different terms, provided the original work is properly cited, appropriate credit is given, any changes made indicated, and the use is non-commercial. See: http://creativecommons.org/licenses/by-nc/4.0/.

\section{ORCID iDs}

Jens Eg Nørgaard http://orcid.org/0000-0002-5256-6108

Stig Andersen http://orcid.org/0000-0003-3632-5213
Jesper Ryg http://orcid.org/0000-0002-8641-3062

\section{REFERENCES}

1 Vellas BJ, Wayne SJ, Romero LJ, et al. Fear of falling and restriction of mobility in elderly fallers. Age Ageing 1997;26:189-93.

2 Cumming RG, Salkeld G, Thomas M. Nursing home admission. Nursing 2000;55:299-305.

3 Masud T, Morris RO. Epidemiology of falls. Age Ageing 2001;30:3-7.

4 Burns E, Kakara R. Deaths from Falls Among Persons Aged $\geq 65$ Years - United States, 2007-2016. MMWR Morb Mortal Wkly Rep 2018;67:509-14.

5 Skov H, Danielsen S. 8 anbefalinger til forebyggelse af ældres faldulykker - den gode kommunale model. 2nd edn. København: Sund By Netværket, 2016. http://sund-by-net.dk/wp-content/ uploads/2016/10/Faldulykker_SundByNetværk_web.pdf

6 Christensen $\mathrm{K}$, Doblhammer $\overline{\mathrm{G}}$, Rau R, et al. Ageing populations: the challenges ahead. Lancet 2009;374:1196-208.

7 Salomon JA, Wang H, Freeman MK, et al. Healthy life expectancy for 187 countries, 1990-2010: a systematic analysis for the global burden disease study 2010. Lancet 2012;380:2144-62.

8 NICE. NICE clinical guideline - Falls: Assessment and prevention of falls in older people. Natl Inst Heal Care Excell 2013;161:1-315.

9 Lewis J. Expenditure on Healthcare in the UK : 2013, 2015.

10 Sherrington C, Fairhall N, Kwok W, et al. Evidence on physical activity and falls prevention for people aged $65+$ years: systematic review to inform the who guidelines on physical activity and sedentary behaviour. Int J Behav Nutr Phys Act 2020:17:144

11 Centers for Disease Control and Prevention. Preventing falls: a guide to implementing effective community-based fall prevention programs. Natl Cent Inj Prev Control 2015;64.

12 Grabiner MD, Crenshaw JR, Hurt CP, et al. Exercise-based fall prevention: can you be a bit more specific? Exerc Sport Sci Rev 2014:42:161-8

13 Nørgaard JE, Jorgensen MG, Ryg J, et al. Effects of gait adaptability training on falls and fall-related fractures in older adults: a systematic review and meta-analysis. Age Ageing 2021;50:1914-24.

14 Okubo Y, Sturnieks DL, Brodie MA, et al. Effect of reactive balance training involving repeated slips and trips on balance recovery among older adults: a blinded randomized controlled trial. $J$ Gerontol A Biol Sci Med Sci 2019;74:1489-96.

15 Bhatt T, Pai Y-C. Prevention of slip-related backward balance loss: the effect of session intensity and frequency on long-term retention. Arch Phys Med Rehabil 2009;90:34-42.

16 Pai Y-C, Bhatt T, Yang F, et al. Perturbation Training Can Reduce Community-Dwelling Older Adults' Annual Fall Risk: A Randomized Controlled Trial. J Gerontol A Biol Sci Med Sci 2014;69:1586-94.

17 Pai Y-C, Bhatt TS. Repeated-slip training: an emerging paradigm for prevention of slip-related falls among older adults. Phys Ther 2007;87:1478-91.

18 Wang Y, Wang S, Bolton R, et al. Effects of task-specific obstacleinduced trip-perturbation training: proactive and reactive adaptation to reduce fall-risk in community-dwelling older adults. Aging Clin Exp Res 2020;32:893-905.

19 Mansfield A, Wong JS, Bryce J, et al. Does perturbation-based balance training prevent falls? systematic review and meta-analysis of preliminary randomized controlled trials. Phys Ther 2015;95:700-9.

20 Okubo Y, Schoene D, Lord SR. Step training improves reaction time, gait and balance and reduces falls in older people: a systematic review and meta-analysis. Br J Sports Med 2017;51:586-93.

21 Wang T-Y, Bhatt T, Yang F, et al. Generalization of motor adaptation to repeated-slip perturbation across tasks. Neuroscience 2011;180:85-95.

22 Pai Y-C, Yang F, Bhatt T, et al. Learning from laboratory-induced falling: long-term motor retention among older adults. Age 2014;36:1367-76.

23 Karamanidis K, Epro G, Mccrum C. Improving Trip- and SlipResisting skills in older people. Perturbation Dose Matters 2020.

24 König M, Epro G, Seeley J, et al. Retention of improvement in gait stability over 14 weeks due to trip-perturbation training is dependent on perturbation dose. J Biomech 2019;84:243-6.

25 Vogler CM, Menant JC, Sherrington C, et al. Evidence of detraining after 12-week home-based exercise programs designed to reduce fall-risk factors in older people recently discharged from hospital. Arch Phys Med Rehabil 2012;93:1685-91.

26 Rivera-Torres S, Fahey TD, Rivera MA. Adherence to exercise programs in older adults: informative report. Gerontol Geriatr Med 2019;5:233372141882360.

27 Nyman SR, Victor CR. Older people's participation in and engagement with falls prevention interventions in community 
settings: an augment to the Cochrane systematic review. Age Ageing 2012;41:16-23.

28 Mansfield A, Peters AL, Liu BA, et al. Effect of a perturbation-based balance training program on compensatory stepping and grasping reactions in older adults: a randomized controlled trial. Phys Ther 2010;90:476-91.

29 Liu X, Bhatt T, Wang Y, et al. The retention of fall-resisting behavior derived from treadmill slip-perturbation training in communitydwelling older adults. Geroscience. 2021;43:913-926.

30 Lee A, Bhatt T, Liu X, et al. Can treadmill slip-perturbation training reduce longer-term fall risk upon overground slip exposure? J Appl Biomech 2020;36:298-306.

31 Rosenblatt NJ, Marone J, Grabiner MD. Preventing trip-related falls by community-dwelling adults: a prospective study. J Am Geriatr Soc 2013:61:1629-31.

32 Lurie JD, Zagaria AB, Ellis L, et al. Surface perturbation training to prevent falls in older adults: a highly pragmatic, randomized controlled trial. Phys Ther 2020;100:1153-62.

33 Berg WP, Alessio HM, Mills EM, et al. Circumstances and consequences of falls in independent community-dwelling older adults. Age Ageing 1997;26:261-8.

34 Luukinen $\mathrm{H}$, Herala M, Koski K, et al. Fracture risk associated with a fall according to type of fall among the elderly. Osteoporos Int 2000;11:631-4

35 Liu X, Bhatt T, Pai Y-CC. Intensity and generalization of treadmill slip training: high or low, progressive increase or decrease? J Biomech 2016;49:135-40.

36 Karamanidis K, Epro G, McCrum C, et al. Improving Trip- and SlipResisting skills in older people: perturbation dose matters. Exerc Sport Sci Rev 2020;48:40-7.

37 Grabiner MD, Bareither ML, Gatts S, et al. Task-specific training reduces trip-related fall risk in women. Med Sci Sports Exerc 2012;44:2410-4.

38 Allin LJ, Brolinson PG, Beach BM, et al. Perturbation-based balance training targeting both slip- and trip-induced falls among older adults: a randomized controlled trial. BMC Geriatr 2020;20:1-13.

39 Gerards MHG, McCrum C, Mansfield A, et al. Perturbation-based balance training for falls reduction among older adults: current evidence and implications for clinical practice. Geriatr Gerontol Int 2017;17:2294-303

40 Hoffmann TC, Glasziou PP, Boutron I. Better reporting of interventions: template for intervention description and replication (TIDieR) checklist and guide. BMJ 2014;348:1-12.

41 Wade E V. The short Orientation-Memory-Concentration test: a study of its reliability and validity. Clin Rehabil 1999;13:164-70.

42 Meyer C, Killeen T, Easthope CS. Familiarization with treadmill walking: how much is enough? Sci Rep 2019;9:1-10.

43 Kang HG, Dingwell JB. Separating the effects of age and walking speed on gait variability. Gait Posture 2008;27:572-7.

44 Row Lazzarini BS, Kataras TJ. Treadmill walking is not equivalent to overground walking for the study of walking smoothness and rhythmicity in older adults. Gait Posture 2016;46:42-6.

45 Okubo Y, Brodie MA, Sturnieks DL. A pilot study of reactive balance training using trips and slips with increasing unpredictability in young and older adults: biomechanical mechanisms, falls and clinical feasibility. Clin Biomech 2019;67:171-9.

46 Takazono PS, Ribeiro de Souza C, Ávila de Oliveira J, et al. High contextual interference in perturbation-based balance training leads to persistent and generalizable stability gains of compensatory limb movements. Exp Brain Res 2020;238:1249-63.

47 McCrum C, Gerards MHG, Karamanidis K. A systematic review of gait perturbation paradigms for improving reactive stepping responses and falls risk among healthy older adults. Eur Rev Aging Phys Act 2017;14:1-11.

48 Bhatt T, Yang F, Pai Y-C. Learning to resist gait-slip falls: long-term retention in community-dwelling older adults. Arch Phys Med Rehabil 2012;93:557-64.

49 Wang $\mathrm{Y}$, Wang S, Lee A, et al. Treadmill-gait slip training in community-dwelling older adults: mechanisms of immediate adaptation for a progressive ascending-mixed-intensity protocol. Exp Brain Res 2019;237:2305-17.

50 Witham MD, Stott DJ. Conducting and reporting trials for older people. Age Ageing 2017;46:889-94.

51 Lamb SE, Jørstad-Stein EC, Hauer K, et al. Development of a common outcome data set for fall injury prevention trials: the prevention of falls network Europe consensus. J Am Geriatr Soc 2005;53:1618-22.

52 Muir-Hunter SW, Wittwer JE. Dual-task testing to predict falls in community-dwelling older adults: a systematic review. Physiotherapy 2016;102:29-40.
53 Lord SR, Fitzpatrick RC. Choice stepping reaction time: a composite measure of falls risk in older people. J Gerontol A Biol Sci Med Sci 2001;56:627-32.

54 Johansson J, Nordström A, Gustafson Y, et al. Increased postural sway during quiet stance as a risk factor for prospective falls in community-dwelling elderly individuals. Age Ageing 2017;46:964-70.

55 Veronese N, Bolzetta F, Toffanello ED, et al. Association between short physical performance battery and falls in older people: the Progetto Veneto Anziani study. Rejuvenation Res 2014;17:276-84.

56 Mirelman A, Herman T, Brozgol M. Executive function and falls in older adults: new findings from a five-year prospective study link fall risk to cognition. PLoS One 2012;7:1-8.

57 Montero-Odasso M, Almeida QJ, Bherer L, et al. Consensus on shared measures of mobility and cognition: from the Canadian Consortium on neurodegeneration in aging (CCNA). The Journals of Gerontology: Series A 2019;74:897-909.

58 Cullen S, Montero-Odasso M, Bherer L, et al. Guidelines for gait assessments in the Canadian Consortium on neurodegeneration in aging (CCNA). Can Geriatr J 2018;21:157-65.

59 Jorgensen MG, Laessoe U, Hendriksen C. Intra-rater reproducibility and validity of Nintendo Wii balance testing in community-dwelling older adults. Eur Geriatr Med 2013;4:S87.

60 Bonnechère $B$, Van Hove $\mathrm{O}$, Jansen $\mathrm{B}$. Validation of the Wii balance board to assess static balance during Dual-task activity in healthy subjects. Med Nov Technol Devices 2019;1:100003.

61 Clark RA, Mentiplay BF, Pua Y-H, et al. Reliability and validity of the Wii balance board for assessment of standing balance: a systematic review. Gait Posture 2018;61:40-54.

62 Jorgensen MG, Paramanathan S, Ryg J. Novel use of the Nintendo Wii board as a measure of reaction time: a study of reproducibility in older and younger adults. BMC Geriatr 2015;15:1-8.

63 Freire AN, Guerra RO, Alvarado B, et al. Validity and reliability of the short physical performance battery in two diverse older adult populations in Quebec and Brazil. J Aging Health 2012:24:863-78.

64 Gullickson T. Review of a compendium of neuropsychological tests: administration, norms, and commentary. Contemp Psychol A J Rev 1992;37:385.

65 Montero-odasso M. Falls and cognition in older persons, 2020.

66 Sánchez-Cubillo I, Periáñez JA, Adrover-Roig D, et al. Construct validity of the TRAIL making test: role of task-switching, working memory, inhibition/interference control, and visuomotor abilities. J Int Neuropsychol Soc 2009;15:438-50.

67 Wade DT, Vergis E. The short Orientation-Memory-Concentration test: a study of its reliability and validity. Clin Rehabil 1999;13:164-70.

68 Sørensen J, Davidsen M, Gudex C, et al. Danish EQ-5D population norms. Scand J Public Health 2009:37:467-74.

69 Yardley L, Beyer N, Hauer K, et al. Development and initial validation of the falls efficacy Scale-International (FES-I). Age Ageing 2005;34:614-9.

70 Mullen SP, Olson EA, Phillips SM, et al. Measuring enjoyment of physical activity in older adults: invariance of the physical activity enjoyment scale (paces) across groups and time. Int J Behav Nutr Phys Act 2011;8:103.

71 Davis JC, Robertson MC, Comans T, et al. Guidelines for conducting and reporting economic evaluation of fall prevention strategies. Osteoporos Int 2011;22:2449-59.

72 Wittrup-Jensen KU, Lauridsen J, Gudex C, et al. Generation of a Danish TTO value set for EQ-5D health states. Scand J Public Health 2009;37:459-66.

73 Harris PA, Taylor R, Minor BL, et al. The REDCap Consortium: building an international community of software platform partners. $J$ Biomed Inform 2019;95:103208.

74 Harris PA, Taylor R, Thielke R, et al. Research electronic data capture (REDCap)--a metadata-driven methodology and workflow process for providing translational research informatics support. J Biomed Inform 2009;42:377-81.

75 Sherrington C, Fairhall NJ, Wallbank GK. Exercise for preventing falls in older people living in the community. Cochrane Database Syst Rev 2019;2019.

76 Robertson MC, Campbell AJ, Herbison P. Statistical analysis of efficacy in falls prevention trials. Journals Gerontol - Ser A Biol Sci Med Sci 2005;60:530-4.

77 McPhate L, Simek EM, Haines TP. Program-related factors are associated with adherence to group exercise interventions for the prevention of falls: a systematic review. $J$ Physiother 2013;59:81-92.

78 Sherrington C, Michaleff ZA, Fairhall N, et al. Exercise to prevent falls in older adults: an updated systematic review and meta-analysis. $\mathrm{Br}$ J Sports Med 2017;51:1749-57. 